IMA Journal of Applied Mathematics (2008) 73, 759-781

doi:10.1093/imamat/hxn015

Advance Access publication on June 23, 2008

\title{
A comparison of periodic travelling wave generation by Robin and Dirichlet boundary conditions in oscillatory reaction-diffusion equations
}

\author{
JONATHAN A. SHERRATT $\dagger$ \\ Department of Mathematics and Maxwell Institute for Mathematical Sciences, \\ Heriot-Watt University, Edinburgh EH14 4AS, UK
}

[Received on 25 April 2007; revised on 23 January 2008; accepted on 29 April 2008]

\begin{abstract}
Periodic travelling waves are an important solution form in oscillatory reaction-diffusion equations. I have shown previously that such waves arise naturally near a boundary at which a Dirichlet condition is applied. This result has applications in ecology, providing a potential explanation for the periodic waves seen in a number of natural populations. However, in ecological applications the Dirichlet boundary condition typically arises as a simple approximation to a more realistic Robin condition. In this paper, I consider the generation of periodic travelling waves by Robin boundary conditions and how the wave amplitude compares with that arising from Dirichlet conditions. I study a ' $\lambda-\omega$ ' system of equations, which is the normal form of an oscillatory reaction-diffusion system with scalar diffusion matrix close to a Hopf bifurcation. I consider a Robin boundary condition close to the Dirichlet limit, with proximity measured by a small parameter $\epsilon$, and I study the equations as a perturbation problem in this small parameter. I show that the perturbation is singular and that although the solution itself changes at $\mathrm{O}(\epsilon)$, the amplitude of the periodic travelling wave which this solution approaches far from the boundary is unchanged at both $\mathrm{O}(\epsilon)$ and $\mathrm{O}\left(\epsilon^{2}\right)$. This provides strong justification for the use of the Dirichlet approximation to the Robin condition when studying periodic travelling wave generation in equations of $\lambda-\omega$ type. Finally, I discuss the ecological applications of the results.
\end{abstract}

Keywords: oscillatory system; perturbation theory; reaction-diffusion; wavetrain.

\section{Introduction}

Oscillatory reaction-diffusion equations are a class of model with important applications in many areas of biology, ranging from calcium signalling in cell biology (Sneyd \& Sherratt, 1997; Timofeeva \& Coombes, 2003) to population dynamics in ecology (Pascual, 1993; Petrovskii et al., 2001; Medvinsky et al., 2002; Garvie, 2007). In one space dimension, the most important solution type for such equations is periodic travelling waves. These are the $1 \mathrm{D}$ version of more complicated $2 \mathrm{D}$ behaviours such as spiral and scroll waves and are also important in their own right. For example, periodic travelling waves have recently been identified in a number of ecological systems (Lambin et al., 1998; Moss et al., 2000; MacKinnon et al., 2001; Bjørnstad et al., 2002; Johnson et al., 2004; Bierman et al., 2006).

Periodic travelling waves arise when oscillatory reaction-diffusion equations are subject to inhomogeneities that drive the system away from spatially uniform oscillations. These may have the form of noise in parameter values (Hagan, 1981; Kopell, 1981; Kay \& Sherratt, 2000) or forcing applied at a boundary of the domain. Both mechanisms have been studied extensively for chains of coupled oscillators (Ermentrout \& Kopell, 1984, 1986; Kopell et al., 1991; Ren \& Ermentrout, 1998), but in oscillatory reaction-diffusion equations, there has been very little work on boundary-driven periodic travelling

\footnotetext{
†Email: jas@ma.hw.ac.uk
} 
waves, despite early work by Auchmuty \& Nicolis (1976) on the Brusselator model with Neumann and Dirichlet boundary conditions.

In a previous paper (Sherratt, 2003), I studied the generation of periodic travelling waves by Dirichlet boundary conditions in a ' $\lambda-\omega$ ' system. These equations are the normal form of an oscillatory reactiondiffusion system with scalar diffusivity close to a supercritical Hopf bifurcation (Hassard et al., 1981; Guckenheimer \& Holmes, 1983) and have the form

$$
\begin{aligned}
& \partial u / \partial t=u_{x x}+\left(1-r^{2}\right) u-\left(\omega_{0}-\omega_{1} r^{2}\right) v, \\
& \partial v / \partial t=v_{x x}+\left(\omega_{0}-\omega_{1} r^{2}\right) u+\left(1-r^{2}\right) v,
\end{aligned}
$$

where $r=\left(u^{2}+v^{2}\right)^{1 / 2}$. In an ecological application, the variables $u$ and $v$ would reflect the deviation of population densities from a coexistence steady state; hence $u$ and $v$ can be positive or negative. The parameters $\omega_{0}$ and $\omega_{1}$ would be functions of ecological parameters that can be derived via the theory of normal forms (Hassard et al., 1981; Guckenheimer \& Holmes, 1983; Sherratt, 2001). In Sherratt (2003), I studied the system (1) on a semi-infinite domain $0 \leqslant x<\infty$, subject to the boundary condition $u=v=0$ at $x=0$. I showed that the long-term solution has a simple analytical form (given below) and that this solution approaches a periodic travelling wave at large $x$.

In many ecological applications, Dirichlet boundary conditions are used as a simplifying approximation, with a more accurate boundary condition being of Robin (mixed) type. The derivation of such a Robin condition was first presented by Ludwig et al. (1979). Suppose that a population of density $W(X, T)$ occupies the region $X>0$, satisfying the equation $W_{T}=D W_{X X}+f(W)$. Suppose further that $X<0$ is a hostile environment on which $W$ satisfies $W_{T}=D W_{X X}-\eta W$. This equation has a unique (up to scaling) bounded equilibrium solution on $X<0$, and matching densities and fluxes at $X=0$ yields

$$
(D / \eta)^{1 / 2} W_{X}=W .
$$

Since the $X<0$ region is hostile, one expects $\eta$ to be a large parameter, so that the Dirichlet condition $W=0$ is a natural approximation at the boundary. In practice, almost all studies use the approximate Dirichlet condition rather than the Robin condition; for recent exceptions, see Cantrell et al. (1998, 2002).

In this paper, I investigate the way in which Robin and Dirichlet boundary conditions differ in terms of the generation of periodic travelling waves. Specifically, I consider (1) on $0 \leqslant x<\infty$, subject to

$$
\epsilon u_{x}=u, \quad \epsilon v_{x}=v \quad \text { at } x=0,
$$

with $\epsilon \ll 1$. This is not directly analogous to (2), since $W$ is a population density, whereas in ecological applications, $u$ and $v$ would correspond to the difference between population density and a non-trivial uniform steady state. The advantage of (3) is that it preserves the circular symmetry inherent in (1) and thus offers mathematical simplification over (2). Therefore, I use (3) as a first stage in the comparison of Robin and Dirichlet boundary conditions. For (1) subject to (3), I will show that although the overall solutions for $u$ and $v$ change at $\mathrm{O}(\epsilon)$, the periodic travelling wave that develops at large $x$ does not change either at order $\epsilon$ or $\epsilon^{2}$. This provides strong justification for the use of the Dirichlet approximation to the Robin condition when studying periodic travelling wave generation in (1).

In Section 2, I give a more detailed introduction to $\lambda-\omega$ systems. In Sections 3 and 4 , I determine the $\mathrm{O}(\epsilon)$ and $\mathrm{O}\left(\epsilon^{2}\right)$ corrections to the solution, showing that in both cases there is no change in the limiting behaviour at large $x$, which is the periodic travelling wave solution. Finally in Section 5, I discuss the implications of my results, focussing in particular on ecological applications. 


\section{2. $\lambda-\omega$ systems}

In their seminal paper on oscillatory reaction-diffusion systems, Kopell \& Howard (1973) showed that any such system with scalar diffusivity has a one-parameter family of periodic travelling waves. They also introduced the $\lambda-\omega$ equations and showed that for (1), the family has the simple form

$$
\begin{aligned}
& u=r^{*} \cos \left[\theta_{0} \pm \sqrt{1-r^{* 2}} x+\left(\omega_{0}-\omega_{1} r^{* 2}\right) t\right], \\
& v=r^{*} \sin \left[\theta_{0} \pm \sqrt{1-r^{* 2}} x+\left(\omega_{0}-\omega_{1} r^{* 2}\right) t\right] .
\end{aligned}
$$

Here, $r^{*}$ is the solution amplitude $\left(0 \leqslant r^{*} \leqslant 1\right)$ and is the most convenient parameter for the wave family. Over the last three decades, the simple form of this periodic wave family has provided an invaluable reference point for the study of periodic travelling waves in more general reaction-diffusion systems. This work has focussed in particular on the existence and stability of periodic travelling waves (Ermentrout, 1981; Maginu, 1979, 1981; Kapitula, 1994), other cases with exact solutions (Cope, 1979; Romero et al., 2000) and the generation of periodic travelling waves by environmental heterogeneities (Auchmuty \& Nicolis, 1976; Hagan, 1981; Kopell, 1981; Kay \& Sherratt, 2000; Sherratt, 2003) and behind invasive wavefronts (Sherratt, 1994, 1996; Ermentrout et al., 1997; Petrovskii et al., 1998; Petrovskii \& Malchow, 2000, 2001; Webb \& Sherratt, 2004; Garvie, 2007).

Analytical study of (1) is greatly facilitated by rewriting the equations using $r=\sqrt{u^{2}+v^{2}}$ and $\theta=\tan ^{-1}(v / u)$ as dependent variables. This gives equations of the form

$$
\begin{gathered}
r_{t}=r_{x x}-r \theta_{x}^{2}+r\left(1-r^{2}\right), \\
\theta_{t}=\theta_{x x}+\frac{2 r_{x} \theta_{x}}{r}+\omega_{0}-\omega_{1} r^{2},
\end{gathered}
$$

while the periodic travelling waves are

$$
r=r^{*}, \quad \theta=\theta_{0} \pm \sqrt{1-r^{* 2}} x+\left(\omega_{0}-\omega_{1} r^{* 2}\right) t .
$$

In terms of $r$ and $\theta$, the Robin boundary condition (3) has the form

$$
\epsilon r_{x}=r, \quad \theta_{x}=0 \quad \text { at } x=0 .
$$

Figure 1 illustrates a typical solution of (1) subject to these end conditions; I plot the solutions for $u$, $v, r$ and $\theta_{x}$. Once initial transients have disappeared, the solution has the form of periodic travelling waves moving away from the $x=0$ boundary. The waves appear as sinusoidal oscillations in $u$ and $v$ and correspond to constant values of $r$ and $\theta_{x}$. In fact, numerical solutions suggest that when plotted in terms of $r$ and $\theta_{x}$, the entire long-term solution is a function of $x$ only, independent of time. Substituting $r=R(x)$ and $\theta_{x}=\Psi(x)$ into (1) gives a third-order system of ordinary differential equations for this long-term solution:

$$
\begin{gathered}
R_{x x}+R\left(1-R^{2}-\Psi^{2}\right)=0, \\
\Psi_{x}+2 \Psi R_{x} / R+\omega_{1} A^{2}-\omega_{1} R^{2}=0 .
\end{gathered}
$$

Here, $A$ is a constant of integration, into which the parameter $\omega_{0}$ has been incorporated; a periodic travelling wave satisfying these equations must have amplitude $A$. The boundary condition (6) implies that

$$
\epsilon R_{x}=R \quad \text { and } \quad \Psi=0 \quad \text { at } x=0 .
$$



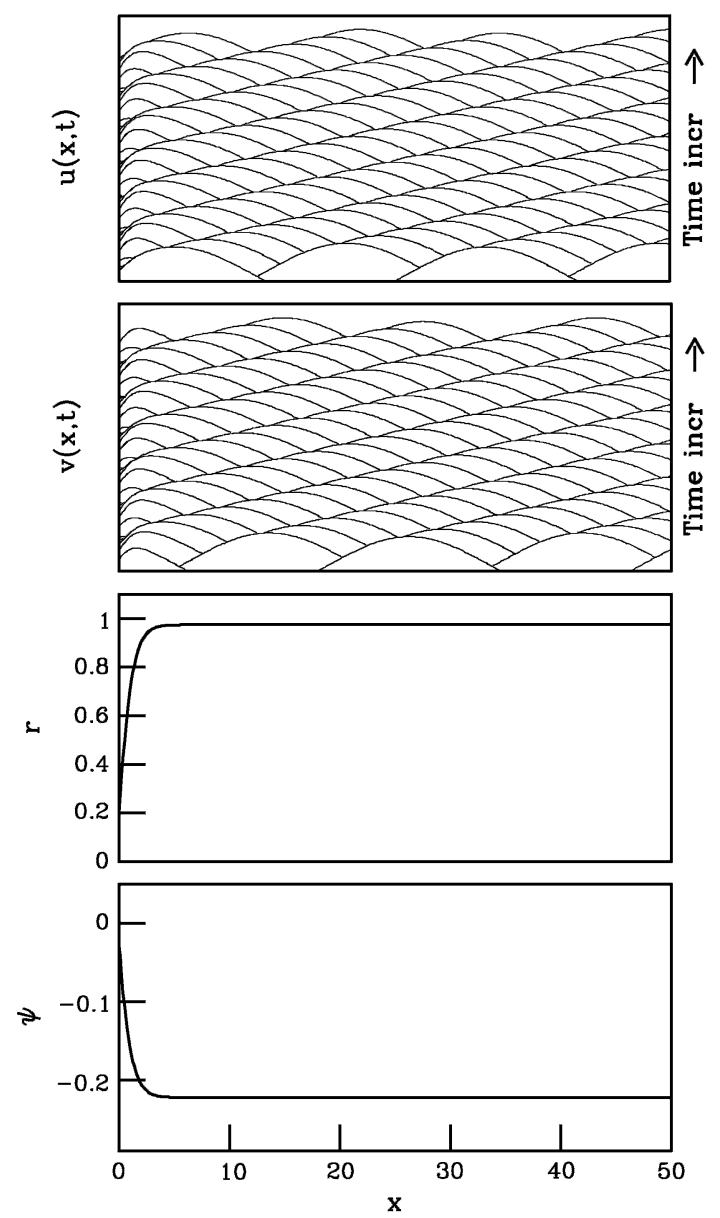

FIG. 1. An illustration of periodic travelling wave generation in the $\lambda-\omega$ system of equations (1) subject to the Robin boundary condition (6) at $x=0$. I show space-time plots for $u$ and $v$, in which a series of solutions are plotted at equally spaced times, with vertical separation corresponding to the time interval. The solution has the form of periodic travelling waves moving away from the $x=0$ boundary. I also plot the long-term solutions for the amplitude $r$ and phase gradient $\psi$. These both evolve to a steady state, independent of time. The parameter values are $\omega_{0}=1.5, \omega_{1}=0.5$ and $\epsilon=0.3$, and the solution is solved on the spatial domain $0<x<250$ with zero-flux boundary conditions at $x=250$, to replicate a semi-infinite domain. The initial conditions for $u$ and $v$ are given by randomly generated values between -1 and 1 . The solutions for $r$ and $\psi$ are plotted at $t=2500$, while for $u$ and $v$, I plot 60 solutions at equally spaced times in the period $2300<t<2500$. The equations were solved numerically using a semi-implicit Crank-Nicolson method.

Since we are looking for a solution that approaches a periodic travelling wave away from the $x=0$ boundary, we also require

$$
R \rightarrow A \quad \text { and } \quad \Psi \rightarrow \sqrt{1-A^{2}} \quad \text { as } x \rightarrow \infty .
$$

The amplitude $A$ will depend on the parameters $\omega_{1}$ and $\epsilon$; Fig. 2 illustrates this dependence, determined from numerical solutions of (1). Note in particular that as $\epsilon$ increases from zero, the wave amplitude $A$ gradually increases. 


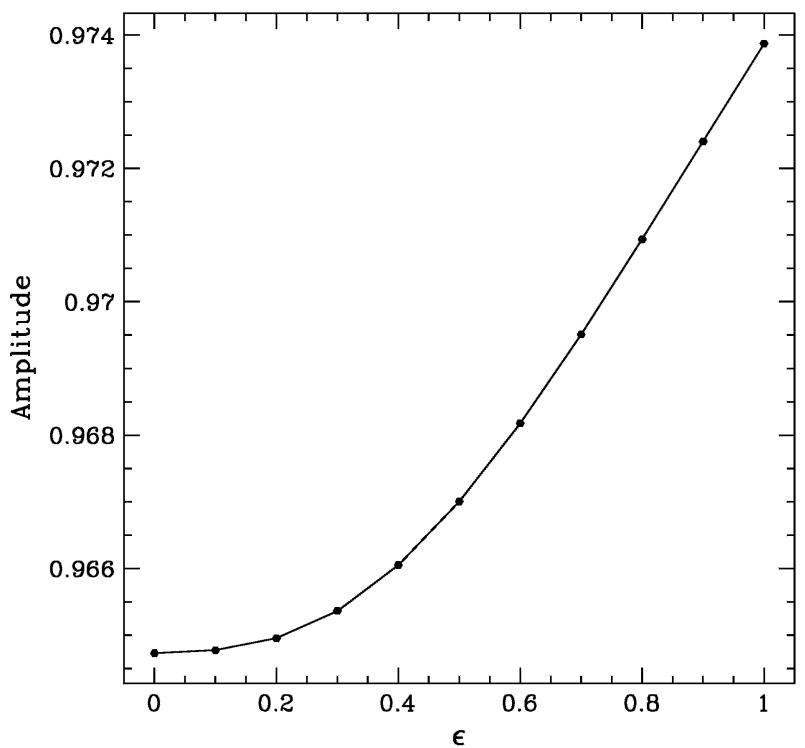

FIG. 2. An illustration of the variation with $\epsilon$ of the amplitude of periodic travelling waves that form away from the $x=0$ boundary in (1) subject to (6). The amplitude is calculated from numerical solutions of $(1,6)$ on a large domain. The parameter values are $\omega_{0}=1.5$ and $\omega_{1}=0.6$.

In Sherratt (2003), I showed that in the case of $\epsilon=0$ (Dirichlet boundary condition), (7-9) has the exact solution

$$
\begin{gathered}
R(x)=R_{0}(x) \equiv a \tanh (x / \sqrt{2}), \\
\Psi(x)=\Psi_{0}(x) \equiv-\operatorname{sign}\left(\omega_{1}\right) \sqrt{1-a^{2}} \tanh (x / \sqrt{2}),
\end{gathered}
$$

with

$$
a=\left.A\right|_{\epsilon=0}=\left\{\frac{1}{2}\left[1+\sqrt{1+\frac{8}{9} \omega_{1}^{2}}\right]\right\}^{-1 / 2} .
$$

This exact solution raises the possibility of determining an approximate form for the solution and in particular the wave amplitude $A$, when $\epsilon$ is small but non-zero.

\section{Solution for small $\epsilon$}

\subsection{Special case of $\omega_{1}=0$}

I begin by considering the special case of $\omega_{1}=0$, which implies that $a=1$. Then, (7-9) can be solved exactly, giving the solution

$$
\begin{gathered}
R=\tanh \left[\left(x+x_{0}\right) / \sqrt{2}\right] \text { and } \phi \equiv 0, \\
\text { where } x_{0}=\frac{1}{\sqrt{2}} \log \left[\sqrt{2} \epsilon+\sqrt{1+2 \epsilon^{2}}\right]=\epsilon-\frac{2}{3} \epsilon^{3}+\mathrm{O}\left(\epsilon^{5}\right) .
\end{gathered}
$$


Therefore in this case, $A \equiv 1$, independent of $\epsilon$, so that the periodic travelling wave generated by the boundary conditions has the degenerate form of a spatially uniform oscillation.

In the subsequent calculations, I assume that $\omega_{1} \neq 0$, so that $a<1$.

\subsection{Formulation of the problem}

Some algebraic simplification in the study of (7) is given by using $y=x / \sqrt{2}$ as independent variable and by substituting $\psi=-\operatorname{sign}\left(\omega_{1}\right) \phi$ and $\left|\omega_{1}\right|=3 \sqrt{1-a^{2}} /\left(a^{2} \sqrt{2}\right)$. Recall that $A$ is the amplitude of the periodic travelling wave solution, while $a$ is the corresponding amplitude when $\epsilon=0$. These substitutions give

$$
\begin{gathered}
\mathrm{d}^{2} R / \mathrm{d} y^{2}+2 R\left(1-R^{2}-\phi^{2}\right)=0, \\
\mathrm{~d} \phi / \mathrm{d} y+2(\phi / R) \mathrm{d} R / \mathrm{d} y-3 \sqrt{1-a^{2}} A^{2} / a^{2}+3 \sqrt{1-a^{2}} R^{2} / a^{2}=0,
\end{gathered}
$$

while the boundary conditions become

$$
\begin{array}{ll}
\epsilon \mathrm{d} R / \mathrm{d} y=R \sqrt{2} \text { and } \phi=0 & \text { at } y=0, \\
R \rightarrow A \text { and } \phi \rightarrow \sqrt{1-A^{2}} & \text { as } y \rightarrow \infty .
\end{array}
$$

For small $\epsilon$, one can investigate solutions of (12) subject to (13) in the usual way, by expanding $R, \psi$ and $A$ as power series in $\epsilon$ :

$$
\begin{gathered}
R=a \tanh y+\epsilon R_{1}+\epsilon^{2} R_{2}+\cdots, \\
\phi=\sqrt{1-a^{2}} \tanh y+\epsilon \phi_{1}+\epsilon^{2} \phi_{2}+\cdots, \\
A=a+\epsilon A_{1}+\epsilon^{2} A_{2}+\cdots .
\end{gathered}
$$

My aim is to determine the corrections $A_{i}$ to the wave amplitude; to do this it is necessary to find the corresponding solutions $R_{i}$ and $\phi_{i}$.

\subsection{Order $\epsilon$ solution}

Substituting (14) into $(12,13)$ and equating coefficients of $\epsilon$ gives

$$
\begin{aligned}
& \mathrm{d}^{2} R_{1} / \mathrm{d} y^{2}+2 R_{1}\left[1-\left(1+2 a^{2}\right) \tanh ^{2} y\right]-4 a \sqrt{1-a^{2}} \tanh ^{2} y \phi_{1}=0, \\
& a \tanh y \mathrm{~d} \phi_{1} / \mathrm{d} y+2 a \operatorname{sech}^{2} y \phi_{1}+2 \sqrt{1-a^{2}}\left[\tanh y \mathrm{~d} R_{1} / \mathrm{d} y\right. \\
& \left.+\left(4 \tanh ^{2} y-1\right) R_{1}-3 A_{1} \tanh y\right]=0 .
\end{aligned}
$$

These equations can be converted into a single third-order equation for $R_{1}$ by differentiating (15a) with respect to $y$ and eliminating $\phi_{1}$, giving

$$
\begin{aligned}
& \mathrm{d}^{3} R_{1} / \mathrm{d} y^{3}+2\left(\mathrm{~d} R_{1} / \mathrm{d} y\right)\left[1-3\left(2 a^{2}-1\right) \tanh ^{2} y\right] \\
& \quad-12 R_{1}\left[\tanh y \operatorname{sech}^{2} y-2\left(1-a^{2}\right) \tanh ^{3} y\right]=g_{1}(y) \equiv 24\left(1-a^{2}\right) A_{1} \tanh ^{2} y .
\end{aligned}
$$

I am looking for solutions of this equation subject to $R_{1}=a / \sqrt{2}$ at $y=0$ and $R_{1} \rightarrow A_{1}$ as $y \rightarrow \infty$. 
The third-order equation (16) can be reduced to second order using the derivative of the leadingorder solution (10). Substituting $R_{1}(y)=\operatorname{sech}^{2} y \Upsilon(y)$ gives

$$
\mathrm{d}^{3} \Upsilon / \mathrm{d} y^{3}-6 \tanh y \mathrm{~d}^{2} \Upsilon / \mathrm{d} y^{2}+\left[12\left(2-a^{2}\right) \tanh ^{2} y-4\right] \mathrm{d} \Upsilon / \mathrm{d} y=g_{1}(y) \cosh ^{2} y .
$$

Further substitution reduces the homogeneous equation corresponding to (17) to a hypergeometric equation. Specifically, I set

$$
w=(\mathrm{d} \Upsilon / \mathrm{d} y) \cosh ^{p} y \quad \text { and } \quad \xi=(1+\tanh y) / 2 \text { with } p=-3+\sqrt{12 a^{2}-11} .
$$

This substitution is adapted from Section 2.1.2.227 of Polyanin \& Zaitsev (2003); after some algebraic simplification, it reduces the homogeneous version of (17) to

$$
\xi(\xi-1) \mathrm{d}^{2} w / \mathrm{d} \xi^{2}+(p+4)(2 \xi-1) \mathrm{d} w / \mathrm{d} \xi+(p+4) w=0 .
$$

Note that for some values of the parameter $a, p$ and hence the solution $w$ are complex. Linearly independent solutions of (19) are given by

$$
w=F(\alpha, \beta, \gamma ; \xi) \quad \text { and } \quad w=F(\alpha, \beta, \gamma ; 1-\xi),
$$

where

$$
\begin{gathered}
\alpha+\beta=2 p+7=1+2 \sqrt{12 a^{2}-11}, \\
\alpha \beta=\gamma=p+4=1+\sqrt{12 a^{2}-11}
\end{gathered}
$$

(Abramowitz \& Stegun, 1964, Section 15.5; Gradshteyn \& Ryzhik, 2000, Section 9.153.7).

The general solution for $\Upsilon(y)$ and hence $R_{1}(y)$ then follows in the standard way (Polyanin \& Zaitsev, 2003, Section 0.2.1-6):

$$
\begin{aligned}
R_{1}(y)= & K_{w} \operatorname{sech}^{2} y\left[\int_{y_{1}=0}^{y_{1}=y} Y^{-}\left(y_{1}\right) \int_{y_{2}=y_{1}}^{y_{2}=\infty} \frac{Y^{+}\left(y_{2}\right) g_{1}\left(y_{2}\right)}{\cosh ^{4} y_{2}} \mathrm{~d} y_{2} \mathrm{~d} y_{1}\right. \\
& \left.-\int_{y_{1}=0}^{y_{1}=y} Y^{+}\left(y_{1}\right) \int_{y_{2}=y_{1}}^{y_{2}=\infty} \frac{Y^{-}\left(y_{2}\right) g_{1}\left(y_{2}\right)}{\cosh ^{4} y_{2}} \mathrm{~d} y_{2} \mathrm{~d} y_{1}\right] \\
& +C_{1,1} \operatorname{sech}^{2} y \int_{y_{1}=0}^{y_{1}=y} Y^{+}\left(y_{1}\right) \mathrm{d} y_{1}+C_{2,1} \operatorname{sech}^{2} y \int_{y_{1}=0}^{y_{1}=y} Y^{-}\left(y_{1}\right) \mathrm{d} y_{1}+C_{3,1} \operatorname{sech}^{2} y,
\end{aligned}
$$

where

$$
Y^{ \pm}(y)=\operatorname{Re}\left[\operatorname{sech}^{p} y F(\alpha, \beta, \gamma,(1 \pm \tanh y) / 2)\right] .
$$

Here, $F(\cdot, \cdot, \cdot, \cdot)$ is the hypergeometric function. The function $g_{1}(y)$ is defined in (16), and the constant $K_{w}$ is defined by $Y^{+}(y) Y^{-^{\prime}}(y)-Y^{-}(y) Y^{+^{\prime}}(y)=K_{w} \cosh ^{6} y . K_{w}$ is a complicated function of $a$ and for brevity I omit its exact form, which is not required in the subsequent calculations. The various limits of integration are chosen to simplify subsequent calculations. In Appendix A, I derive the behaviour of (22) as $y \rightarrow \infty$. This shows that the condition $R_{1} \rightarrow A_{1}$ as $y \rightarrow \infty$ is satisfied if and only if $C_{1,1}=C_{2,1}=0$. 
To consider the boundary conditions at $y=0$, it is necessary to investigate the behaviour of the solution (22) near $y=0$. I define

$$
\begin{aligned}
H_{1}= & K_{w}\left[Y^{-}(0) \int_{y_{2}=0}^{y_{2}=\infty} \frac{Y^{+}\left(y_{2}\right) g_{1}\left(y_{2}\right)}{\cosh ^{4} y_{2}} \mathrm{~d} y_{2}-Y^{+}(0) \int_{y_{2}=0}^{y_{2}=\infty} \frac{Y^{-}\left(y_{2}\right) g_{1}\left(y_{2}\right)}{\cosh ^{4} y_{2}} \mathrm{~d} y_{2}\right] \\
= & 24\left(1-a^{2}\right) A_{1} K_{w} \operatorname{Re}\left[F\left(\alpha, \beta, \gamma ; \frac{1}{2}\right)\right] \\
& \times \operatorname{Re} \int_{y=0}^{y=\infty}\left[F\left(\alpha, \beta, \gamma ; \frac{1+\tanh y}{2}\right)-F\left(\alpha, \beta, \gamma ; \frac{1-\tanh y}{2}\right)\right] \tanh ^{2} y \operatorname{sech}^{4+p} y \mathrm{~d} y, \\
I_{1}= & \left.K_{w} \frac{\mathrm{d}}{\mathrm{d} y_{1}}\left[Y^{-}\left(y_{1}\right) \int_{y_{2}=y_{1}}^{y_{2}=\infty} \frac{Y^{+}\left(y_{2}\right) g_{1}\left(y_{2}\right)}{\cosh ^{4} y_{2}} \mathrm{~d} y_{2}-Y^{+}\left(y_{1}\right) \int_{y_{2}=y_{1}}^{y_{2}=\infty} \frac{Y^{-}\left(y_{2}\right) g_{1}\left(y_{2}\right)}{\cosh ^{4} y_{2}} \mathrm{~d} y_{2}\right]\right|_{y_{1}=0} \\
= & 24\left(1-a^{2}\right) A_{1} K_{w} \operatorname{Re}\left[F^{\prime}\left(\alpha, \beta, \gamma ; \frac{1}{2}\right)\right] \\
& \times \operatorname{Re} \int_{y=0}^{y=\infty}\left[F\left(\alpha, \beta, \gamma ; \frac{1+\tanh y}{2}\right)+F\left(\alpha, \beta, \gamma ; \frac{1-\tanh _{2}}{2}\right)\right] \tanh ^{2} y \operatorname{sech}^{4+p} y \mathrm{~d} y .
\end{aligned}
$$

Expanding the coefficients of (16) as power series in $y$ then implies that near $y=0$

$$
R_{1}(y)=C_{3,1}+H_{1} y+\left(\frac{1}{2} I_{1}-C_{3,1}\right) y^{2}-\frac{1}{3} H_{1} y^{3}+\left[\frac{2}{3} C_{3,1}-\frac{1}{12} I_{1}\right] y^{4}+\mathrm{O}\left(y^{5}\right)
$$

using (22). Equation (15a) then implies that

$$
\phi_{1}(y)=\frac{I_{1}}{4 a \sqrt{1-a^{2}}} \frac{1}{y^{2}}+\frac{C_{3,1} \sqrt{1-a^{2}}}{a}+\mathrm{O}(y)
$$

for small $y$.

There are two undetermined constants in the solution I have derived: $C_{3,1}$ and $A_{1}$. One anticipates that these constants will be determined by the boundary conditions $R_{1}(0)=a / \sqrt{2}$ and $\phi_{1}(0)=0$; however, the expansions (26) and (27) imply that the boundary conditions cannot both be satisfied for any values of $C_{3,1}$ and $A_{1}$. This suggests that an inner (boundary) layer solution is required near $y=0$, and I now consider the form of such an inner solution.

\subsection{Leading-order inner solution and matching}

The appropriate inner equations are given by re-scaling $y, R$ and $\phi$ near $y=0$ :

$$
\xi=y / \epsilon, \quad \tilde{R}=R / \epsilon, \quad \tilde{\phi}=\phi / \epsilon .
$$

Substituting these re-scalings into (12) gives

$$
\begin{gathered}
\mathrm{d}^{2} \tilde{R} / \mathrm{d} \xi^{2}+2 \epsilon^{2} \tilde{R}-2 \epsilon^{4} \tilde{R}\left(\tilde{R}^{2}+\tilde{\phi}^{2}\right)=0 \\
\frac{a^{2} \mathrm{~d}\left(\tilde{R}^{2} \tilde{\phi}\right) / \mathrm{d} \xi}{3 \sqrt{1-a^{2}}}-\tilde{R}^{2}\left[a^{2}+2 A_{1} \epsilon a+\left(A_{1}^{2}+2 a A_{2}\right) \epsilon^{2}\right]+\epsilon^{2} \tilde{R}^{4}=\mathrm{O}\left(\epsilon^{3}\right) .
\end{gathered}
$$


In the usual way, I expand $\tilde{R}$ and $\tilde{\phi}$ as power series in $\epsilon$ :

$$
\begin{aligned}
& \tilde{R}=\tilde{R}_{0}+\epsilon \tilde{R}_{1}+\epsilon^{2} \tilde{R}_{2}, \\
& \tilde{\phi}=\tilde{\phi}_{0}+\epsilon \tilde{\phi}_{1}+\epsilon^{2} \tilde{\phi}_{2}
\end{aligned}
$$

and substituting these into (28) and (13a) gives to leading order

$$
\begin{gathered}
\mathrm{d}^{2} \tilde{R}_{0} / \mathrm{d} \xi^{2}=0, \\
\mathrm{~d}\left(\tilde{R}_{0}^{2} \tilde{\phi}_{0}\right) / \mathrm{d} \xi=3 \sqrt{1-a^{2}} \tilde{R}_{0}^{2}
\end{gathered}
$$

subject to $\mathrm{d} \tilde{R}_{0} / \mathrm{d} \xi=\tilde{R}_{0} \sqrt{2}$ and $\tilde{\phi}_{0}=0$ at $\xi=0$. The solution of these equations is

$$
\tilde{R}_{0}=D_{1}(1+\xi \sqrt{2}), \quad \tilde{\phi}_{0}=\sqrt{\left(1-a^{2}\right) / 2}\left[1+\xi \sqrt{2}-1 /(1+\xi \sqrt{2})^{2}\right],
$$

where $D_{1}$ is a constant of integration. To match this leading-order inner solution with the outer solutions $R_{0}+\epsilon R_{1}$ and $\phi_{0}+\epsilon \phi_{1}$, it is necessary to expand the inner solutions for $\xi \rightarrow \infty$ and to use the expansions (26) and (27) near $y=0$. I express these expansions in terms of the intermediate variable $z=y / v(\epsilon)=\epsilon \xi / v(\epsilon)$, where $1 \gg v(\epsilon) \gg \epsilon$. Then, the conditions for matching are

$$
\overbrace{a v z+\mathrm{O}\left(v^{3}\right)}^{R_{0}}+\overbrace{\epsilon\left[C_{3,1}+\mathrm{O}(v)\right]}^{\epsilon R_{1}}=\overbrace{\epsilon D_{1}(1+\sqrt{2} v z / \epsilon)}^{\epsilon \tilde{R}_{0}}
$$

and

$$
\begin{aligned}
& \underbrace{\sqrt{1-a^{2}} \nu z+\mathrm{O}\left(v^{3}\right)}_{\phi_{0}}+\underbrace{\frac{\epsilon I_{1}}{4 a \sqrt{1-a^{2}} v^{2} z^{2}}+\epsilon \frac{C_{3,1} \sqrt{1-a^{2}}}{a}+\mathrm{O}(\epsilon \nu)}_{\epsilon \phi_{1}} \\
& =\underbrace{\epsilon \sqrt{\left(1-a^{2}\right) / 2}\left[\sqrt{2} \nu z / \epsilon+1+\left(\epsilon^{2} / \nu^{2}\right)\right]}_{\epsilon \tilde{\phi}_{0}} .
\end{aligned}
$$

These conditions are satisfied provided that $D_{1}=C_{3,1}=a / \sqrt{2}$ and $I_{1}=0$. In Appendix B, I show that

$$
\int_{y=0}^{y=\infty}\left[F\left(\alpha, \beta, \gamma ; \frac{1+\tanh y}{2}\right)+F\left(\alpha, \beta, \gamma ; \frac{1-\tanh y}{2}\right)\right] \tanh ^{2} y \operatorname{sech}^{4+p} y \mathrm{~d} y
$$

and $F^{\prime}\left(\alpha, \beta, \gamma ; \frac{1}{2}\right)$ both have non-zero real part for all $a \in(0,1)$. Therefore, the condition $I_{1}=0$ implies that $A_{1}=0$.

With these values for the outstanding constants of integration, the leading-order correction to the solutions when $\epsilon \neq 0$ is given by the combination of $R_{1}$ and $\tilde{R}_{0}$, and $\phi_{1}$ and $\tilde{\phi}_{0}$; composite solutions can easily be determined. But crucially I have shown that $A_{1}=0$, so that this leading-order correction does not affect the periodic wave amplitude $A$, which is unchanged at this order.

\section{Higher-order terms}

I have shown that the periodic travelling wave amplitude $A$ is the same for the Robin (6) and Dirichlet $\left(r=\theta_{x}=0\right.$ at $x=0$ ) boundary conditions, at order $\epsilon$. To determine the next correction $\epsilon^{2} A_{2}$ to $A$, it is necessary to calculate higher-order terms in both the outer and the inner solutions. 


\subsection{Higher-order terms in the outer solution}

Equating coefficients of $\epsilon^{2}$ in $(12,13)$ gives equations for $R_{2}(y)$ and $\phi_{2}(y)$. These can be reduced to a single third-order equation for $R_{2}$ in a manner directly analogous to that used in the calculation of $R_{1}$; the resulting equation is

$$
\begin{aligned}
& \mathrm{d}^{3} R_{2} / \mathrm{d} y^{3}+2\left(\mathrm{~d} R_{2} / \mathrm{d} y\right)\left[1-3\left(2 a^{2}-1\right) \tanh ^{2} y\right]-12 R_{2}\left[\tanh y \operatorname{sech}^{2} y-2\left(1-a^{2}\right) \tanh ^{3} y\right] \\
& \quad=g_{2}(y) \equiv 24\left(1-a^{2}\right) A_{2} \tanh ^{2} y+6 a\left(a^{2}-3\right) \operatorname{sech}^{4} y+3 a\left(7-2 a^{2}\right) \operatorname{sech}^{6} y .
\end{aligned}
$$

This is the same as (16) except for the difference in the right-hand side, and the solution of the equation proceeds in exactly the same way as for (16), giving

$$
\begin{aligned}
R_{2}(y)= & K_{w} \operatorname{sech}^{2} y\left[\int_{y_{1}=0}^{y_{1}=y} Y^{-}\left(y_{1}\right) \int_{y_{2}=y_{1}}^{y_{2}=\infty} \frac{Y^{+}\left(y_{2}\right) g_{2}\left(y_{2}\right)}{\cosh ^{4} y_{2}} \mathrm{~d} y_{2} \mathrm{~d} y_{1}\right. \\
& \left.\quad-\int_{y_{1}=0}^{y_{1}=y} Y^{+}\left(y_{1}\right) \int_{y_{2}=y_{1}}^{y_{2}=\infty} \frac{Y^{-}\left(y_{2}\right) g_{2}\left(y_{2}\right)}{\cosh ^{4} y_{2}} \mathrm{~d} y_{2} \mathrm{~d} y_{1}\right] \\
& +C_{1,2} \operatorname{sech}^{2} y \int_{y_{1}=0}^{y_{1}=y} Y^{+}\left(y_{1}\right) \mathrm{d} y_{1}+C_{2,2} \operatorname{sech}^{2} y \int_{y_{1}=0}^{y_{1}=y} Y^{-}\left(y_{1}\right) \mathrm{d} y_{1}+C_{3,2} \operatorname{sech}^{2} y .
\end{aligned}
$$

I show in Appendix A that the condition $R_{2} \rightarrow A_{2}$ as $y \rightarrow \infty$ is satisfied if and only if $C_{1,2}=C_{2,2}=0$.

For matching, we will require the behaviour of this solution near $y=0$. I define

$$
\begin{gathered}
H_{2}=K_{w}\left[Y^{-}(0) \int_{y_{2}=0}^{y_{2}=\infty} \frac{Y^{+}\left(y_{2}\right) g_{2}\left(y_{2}\right)}{\cosh ^{4} y_{2}} \mathrm{~d} y_{2}-Y^{+}(0) \int_{y_{2}=0}^{y_{2}=\infty} \frac{Y^{-}\left(y_{2}\right) g_{2}\left(y_{2}\right)}{\cosh ^{4} y_{2}} \mathrm{~d} y_{2}\right], \\
I_{2}=\left.K_{w} \frac{\mathrm{d}}{\mathrm{d} y_{1}}\left[Y^{-}\left(y_{1}\right) \int_{y_{2}=y_{1}}^{y_{2}=\infty} \frac{Y^{+}\left(y_{2}\right) g_{2}\left(y_{2}\right)}{\cosh ^{4} y_{2}} \mathrm{~d} y_{2}-Y^{+}\left(y_{1}\right) \int_{y_{2}=y_{1}}^{y_{2}=\infty} \frac{Y^{-}\left(y_{2}\right) g_{2}\left(y_{2}\right)}{\cosh ^{4} y_{2}} \mathrm{~d} y_{2}\right]\right|_{y_{1}=0} .
\end{gathered}
$$

Expansion of the coefficients of (30) as power series near $y=0$ then gives

$$
\begin{aligned}
R_{2}(y)= & C_{3,2}+H_{2} y+\left(\frac{1}{2} I_{2}-C_{3,2}\right) y^{2}+\left[\frac{1}{2} a-\frac{1}{3} H_{2}\right] y^{3}+\left[\frac{2}{3} C_{3,2}-\frac{1}{12} I_{2}\right] y^{4} \\
& +\left[\left(\frac{1}{5} a^{2}+\frac{2}{15}\right) H_{2}+\frac{2}{5}\left(1-a^{2}\right) A_{2}+\frac{1}{10}\left(a^{3}-5 a\right)\right] y^{5}+\mathrm{O}\left(y^{6}\right) .
\end{aligned}
$$

The solution for $\phi_{2}(y)$ can then be calculated from

$$
\phi_{2}=\frac{\mathrm{d}^{2} R_{2} / \mathrm{d} y^{2}+2 R_{2}\left[1-\left(1+2 a^{2}\right) \tanh ^{2} y\right]-3 a \tanh y \operatorname{sech}^{4} y}{4 a \sqrt{1-a^{2}} \tanh ^{2} y}
$$


which implies

$$
\phi_{2}(y)=\frac{I_{1}}{4 a \sqrt{1-a^{2}}} \frac{1}{y^{2}}+\frac{C_{3,2} \sqrt{1-a^{2}}}{a}+\frac{\sqrt{1-a^{2}}\left(4 A_{2}-a\right)}{2 a} y+\mathrm{O}\left(y^{2}\right) .
$$

\subsection{Higher-order terms in the inner solution}

The expansions (34) and (35) match with higher-order terms in the inner solution. It is necessary to calculate $\tilde{R}_{1}, \tilde{\phi}_{1}, \tilde{R}_{2}$ and $\tilde{\phi}_{2}$ in order to complete the matching. Equating coefficients of $\epsilon$ in (28) gives

$$
\begin{gathered}
\mathrm{d}^{2} \tilde{R}_{1} / \mathrm{d} \xi^{2}=0, \\
(\mathrm{~d} / \mathrm{d} \xi)\left(\tilde{R}_{0}^{2} \tilde{\phi}_{1}+2 \tilde{R}_{0} \tilde{R}_{1} \tilde{\phi}_{0}\right)=6 \sqrt{1-a^{2}} \tilde{R}_{0} \tilde{R}_{1} .
\end{gathered}
$$

These equations are subject to the end conditions $\mathrm{d} \tilde{R}_{1} / \mathrm{d} \xi=\tilde{R}_{1} \sqrt{2}$ and $\tilde{\phi}_{1}=0$ at $\xi=0$. Substituting the leading-order inner solutions (29) into (36) gives the solution

$$
\tilde{R}_{1}=D_{2}(1+\xi \sqrt{2}), \quad \tilde{\phi}_{1}=0
$$

where $D_{2}$ is a constant of integration. Similarly, equating coefficients of $\epsilon^{2}$ in (28) gives

$$
\begin{gathered}
\mathrm{d}^{2} \tilde{R}_{2} / \mathrm{d} \xi^{2}+2 \tilde{R}_{0}=0, \\
(\mathrm{~d} / \mathrm{d} \xi)\left(\tilde{R}_{0}^{2} \tilde{\phi}_{2}+\tilde{R}_{1}^{2} \tilde{\phi}_{0}+2 \tilde{R}_{0} \tilde{R}_{2} \tilde{\phi}_{0}\right)=3 \sqrt{1-a^{2}}\left(\tilde{R}_{1}^{2}+2 \tilde{R}_{0} \tilde{R}_{2}+2 A_{2} \tilde{R}_{0}^{2} / a-\tilde{R}_{0}^{4} / a^{2}\right)
\end{gathered}
$$

with end conditions $\mathrm{d} \tilde{R}_{2} / \mathrm{d} \xi=\tilde{R}_{2} \sqrt{2}$ and $\tilde{\phi}_{2}=0$ at $\xi=0$. Using (29) and (37), this has the solution

$$
\begin{gathered}
\tilde{R}_{2}=D_{3}(1+\xi \sqrt{2})-a\left(\frac{1}{\sqrt{2}} \xi^{2}+\frac{1}{3} \xi^{3}\right), \\
\tilde{\phi}_{2}=\frac{\sqrt{2\left(1-a^{2}\right)} A_{2}}{a}\left[1+\xi \sqrt{2}-\frac{1}{(1+\xi \sqrt{2})^{2}}\right] \\
-\frac{\sqrt{1-a^{2}}}{3(1+\xi \sqrt{2})^{3}}\left[8 \xi^{6}+24 \sqrt{2} \xi^{5}+60 \xi^{4}+48 \sqrt{2} \xi^{3}+54 \xi^{2}+9 \sqrt{2} \xi\right] .
\end{gathered}
$$

\subsection{Higher-order matching}

Expansions of the solutions (37) and (38) near $\xi=\infty$ are straightforward. These expansions together with (34) and (35) give conditions for matching; as in the leading-order matching, I use the intermediate variable $z=y / \nu(\epsilon)=\epsilon \xi / \nu(\epsilon)$, where $1 \gg \nu(\epsilon) \gg \epsilon$. The conditions are

$$
\begin{aligned}
& \overbrace{a\left[v z-\frac{1}{3} v^{3} z^{3}\right]+\mathrm{O}\left(v^{5}\right)}^{R_{0}}+\overbrace{(\epsilon a / \sqrt{2})\left[1-v^{2} z^{2}\right]+\mathrm{O}\left(\epsilon v^{4}\right)}^{\epsilon R_{1}} \\
& +\overbrace{\epsilon^{2}\left[C_{3,2}+H_{2} v z+\left\{\frac{1}{2} I_{2}-C_{3,2}\right\} v^{2} z^{2}\right]+\mathrm{O}\left(\epsilon^{2} v^{3}\right)}^{\epsilon^{2} R_{2}}
\end{aligned}
$$




$$
\begin{aligned}
& =\underbrace{(\epsilon a / \sqrt{2})(1+\sqrt{2} v z / \epsilon)}_{\epsilon \tilde{R}_{0}}+\underbrace{\epsilon^{2} D_{2}(1+\sqrt{2} \nu z / \epsilon)}_{\epsilon^{2} \tilde{R}_{1}} \\
& +\underbrace{\epsilon^{3}\left[D_{3}(1+\sqrt{2} v z / \epsilon)-a\left(\frac{1}{\sqrt{2}} v^{2} z^{2} / \epsilon^{2}+\frac{1}{3} v^{3} z^{3} / \epsilon^{3}\right)\right]}_{\epsilon^{3} \tilde{R}_{2}}
\end{aligned}
$$

and

$$
\begin{aligned}
& \overbrace{\sqrt{1-a^{2}}\left(v z-\frac{1}{3} v^{3} z^{3}\right)+\mathrm{O}\left(v^{5}\right)}^{\phi_{0}}+\overbrace{\epsilon \sqrt{\left(1-a^{2}\right) / 2}\left(1-v^{2} z^{2}\right)+\mathrm{O}\left(\epsilon v^{4}\right)}^{\epsilon \phi_{1}} \\
& +\overbrace{\epsilon^{2}\left[\left\{\frac{I_{2}}{4 a \sqrt{1-a^{2}}}\right\} \frac{1}{v^{2} z^{2}}\right.}^{\epsilon^{2} \text { (continued on next line) }} \\
& \epsilon^{2} \phi_{2} \text { (continued from previous line) } \\
& +\overbrace{\left.\left\{\frac{C_{3,2} \sqrt{1-a^{2}}}{a}+\frac{I_{2}}{6 a \sqrt{1-a^{2}}}\right\}+\left\{\frac{\left(4 A_{2}-a\right) \sqrt{1-a^{2}}}{2 a}\right\} v z\right]+\mathrm{O}\left(\epsilon^{2} v^{2}\right)} \\
& =\underbrace{\epsilon \sqrt{\left(1-a^{2}\right) / 2}[\sqrt{2} v z / \epsilon+1]+\mathrm{O}\left(\epsilon^{3} / \nu^{2}\right)}_{\epsilon \tilde{\phi}_{0}}+\underbrace{0}_{\epsilon^{2} \tilde{\phi}_{1}} \\
& +\underbrace{\epsilon^{3} \sqrt{1-a^{2}}\left[2\left(A_{2} / a\right) \nu z / \epsilon-\left(\frac{1}{3} \nu^{3} z^{3} / \epsilon^{3}+\frac{1}{\sqrt{2}} v^{2} z^{2} / \epsilon^{2}+\frac{1}{2} \nu z / \epsilon\right)\right]+\left(\epsilon^{3}\right)}_{\epsilon^{3} \tilde{\phi}_{2}} .
\end{aligned}
$$

Therefore, the solutions match provided $D_{2}=0, D_{3}=H_{2} / \sqrt{2}, C_{3,2}=0$ and $I_{2}=0$. These conditions determine all the outstanding constants. In particular, the last of the conditions determines $A_{2}$. Since $g_{2}(\cdot)$ is an even function, (33) and (30) give

$$
\begin{aligned}
I_{2}=0 \Leftrightarrow \operatorname{Re}\left[F^{\prime}\left(\alpha, \beta, \gamma ; \frac{1}{2}\right)\right] \operatorname{Re} \int_{-\infty}^{+\infty} F\left(\alpha, \beta, \gamma ; \frac{1+\tanh y}{2}\right) g_{2}(y) \operatorname{sech}^{4+p} y \mathrm{~d} y=0 \\
\Leftrightarrow 24\left(1-a^{2}\right) A_{2} \operatorname{Re} \int_{-\infty}^{+\infty} F\left(\alpha, \beta, \gamma ; \frac{1+\tanh y}{2}\right) \tanh ^{2} y \operatorname{sech}^{4+p} y \mathrm{~d} y \\
=3 a \operatorname{Re}\left[\left(6-2 a^{2}\right) \int_{-\infty}^{+\infty} F\left(\alpha, \beta, \gamma ; \frac{1+\tanh y}{2}\right) \operatorname{sech}^{8+p} y \mathrm{~d} y\right. \\
\left.\quad+\left(2 a^{2}-7\right) \int_{-\infty}^{+\infty} F\left(\alpha, \beta, \gamma ; \frac{1+\tanh y}{2}\right) \operatorname{sech}^{10+p} y \mathrm{~d} y\right] .
\end{aligned}
$$


Here, I am using $\operatorname{Re} F^{\prime}\left(\alpha, \beta, \gamma ; \frac{1}{2}\right) \neq 0$, which is shown in Appendix B. In Appendix $\mathrm{B}$, I also show that the right-hand side of (39) is identically zero, while the integral on the left-hand side has non-zero real part for all $a \in(0,1)$. Therefore, $A_{2}=0$, so that even though the solution for $R$ has a correction at $\mathrm{O}(\epsilon)$, the periodic travelling wave that this solution approaches at large $y$ has no correction at either $\mathrm{O}(\epsilon)$ or $\mathrm{O}\left(\epsilon^{2}\right)$.

\section{Discussion}

There have been relatively few studies of pattern formation on domains with Robin boundary conditions. Some work on spiral waves uses Robin conditions (Golubitsky et al., 2000; Ramos, 2003), which give eigenfunctions with prominent spiral features at a Hopf bifurcation. There have also been some studies of stationary patterns in reaction-diffusion systems with Robin conditions, motivated by applications in physics (Hassan et al., 1994), chemistry and physiology (Wio et al., 1993; von Haeften et al., 1997; Izus et al., 1998) and developmental biology (Dillon et al., 1994; Maini et al., 2007). None of this work involves applications to ecology, despite Robin boundary conditions being very natural in this context, as the appropriate condition when the domain of interest is surrounded by a hostile environment (Ludwig et al., 1979, Section 1; Cantrell et al., 1998). In such applications, the hostility of the environment means that the Robin condition will be close to the Dirichlet limit. In this paper, I have focussed on a reactiondiffusion system of $\lambda-\omega$ form, which is generic because it is the normal form of any oscillatory system with scalar diffusion close to a supercritical Hopf bifurcation. I have shown that Robin and Dirichlet conditions generate a periodic travelling wave of very similar amplitude-much closer than one would expect intuitively. My specific result is that for the boundary condition (3), the amplitude of the periodic travelling wave generated far from the boundary has no correction at either $\mathrm{O}(\epsilon)$ or $\mathrm{O}\left(\epsilon^{2}\right)$, although the solution more generally has a correction at both orders. The complexity of the $\mathrm{O}\left(\epsilon^{2}\right)$ solution means that explicit calculation of the $\mathrm{O}\left(\epsilon^{3}\right)$ correction to the wave amplitude would be extremely laborious but numerical calculations indicate that $A_{3}$ is non-zero. Typical numerical results are illustrated in Fig. 3, and details of the numerical method are given in Appendix C. From the viewpoint of applications,
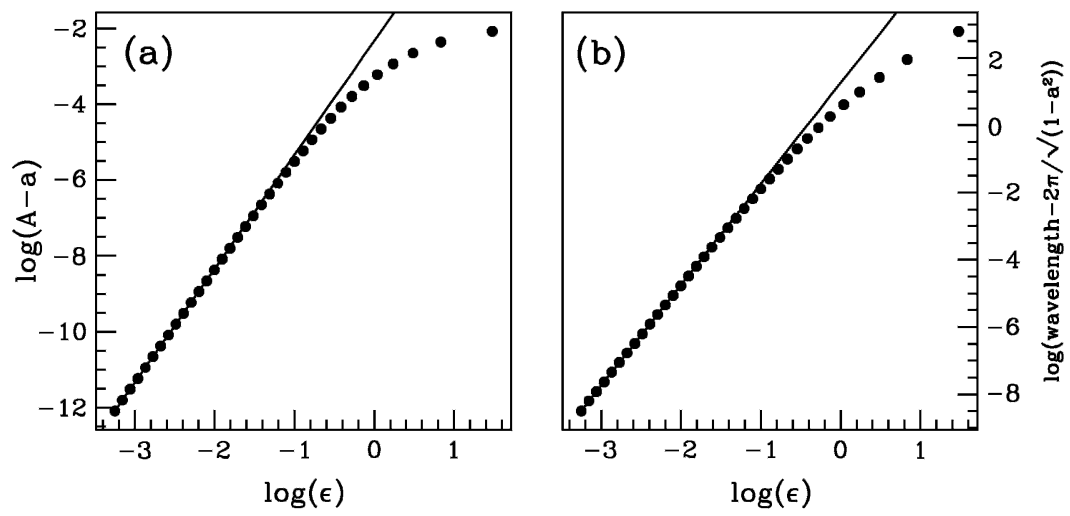

FIG. 3. An illustration of numerical results indicating that $A_{3} \neq 0$, so that there is an $\mathrm{O}\left(\epsilon^{3}\right)$ correction to the periodic travelling wave amplitude. I show (•) (a) $A-a$ and (b) wavelength $-2 \pi / \sqrt{1-a^{2}}$. Both plots are against $\epsilon$ on logarithmic axes for $a=0.85$. Superimposed on these results are lines of slope 3. Details of the numerical method used to calculate $A(\epsilon)$ (and the corresponding wavelength) are given in Appendix C. 
the actual value of $A_{3}$ is not critical: the key result is the absence of $\mathrm{O}(\epsilon)$ and $\mathrm{O}\left(\epsilon^{2}\right)$ corrections to the solution amplitude. Wavelength, time period and wave speed are all simple functions of the amplitude $A$ :

$$
\text { wave speed }=\frac{\omega_{0}-\omega_{1} A^{2}}{\sqrt{1-A^{2}}}, \quad \text { wavelength }=\frac{2 \pi}{\sqrt{1-A^{2}}}, \quad \text { time period }=\frac{2 \pi}{\omega_{0}-\omega_{1} A^{2}} .
$$

Therefore, the corrections to these wave properties are also $\mathrm{O}\left(\epsilon^{3}\right)$. It follows that the Dirichlet boundary condition is a very good approximation indeed to the Robin condition, from the viewpoint of periodic travelling wave generation.

There are two main reasons for investigating the quality of this approximation, rather than just using the Robin condition directly. The first is that the zero density condition is significantly simpler mathematically. Analysis is much easier for the Dirichlet condition (e.g. Sherratt, 2003; Smith et al., 2008); moreover, numerical solution is much more difficult for the Robin condition, especially in two dimensions. Secondly, there are often no quantitative data on the extent of the hostility of the surrounding environment, so that although one anticipates that the Robin condition will be close to the Dirichlet limit, the actual proximity is hard to estimate. For these reasons, it is important to understand the effect of using the Robin rather than zero Dirichlet boundary condition.

I am aware of two examples of periodic travelling waves in ecological field data for which Robin conditions at one boundary provide a plausible explanation. Between 1962 and 1978, a large spatiotemporal data set was gathered on red grouse (Lagopus lagopus scoticus) on Kerloch Moor (NorthEast Scotland) (Watson et al., 1984). Subsequent analysis of the data has revealed periodic travelling waves moving across the domain at a speed of $2-3 \mathrm{~km} / \mathrm{year}$ (Moss et al., 2000). One edge of the study area is bordered by farmland, which is a very hostile environment for red grouse (Piertney et al., 1998). Therefore, a Robin condition is appropriate at this boundary and could be responsible for the observed periodic travelling waves.

The second example concerns field voles (Microtus agrestis) in Kielder Forest (Northern UK). A spatiotemporal field study of these voles has been running for about 20 years and shows that the voles exhibit population cycles that are spatially organized into periodic travelling waves, moving at a speed of 15-20 km/year (Lambin et al., 1988; MacKinnon et al., 2001). The voles are subject to both terrestrial predation (mainly weasels, Mustela nivalis) and avian predation (mainly short-eared owls, Asio flammeus) (Petty et al., 2000). The former will be roughly uniform across the forest, but one expects that the latter will be significantly greater in and around large open spaces, which facilitate hunting. By far the largest such open space is Kielder Water, a very large reservoir in the middle of the forest. The natural boundary condition for voles at the reservoir edge would therefore be of Robin type, corresponding to the rate at which voles are killed at the reservoir edge being proportional to vole density there; the condition would be close to the Dirichlet limit because of the high level of avian predation, which removes $10-15 \%$ of the overall vole population per year (Petty et al., 2000). Therefore, in this case also, the observed periodic waves can be explained by a Robin condition at a boundary of the domain.

There are two main differences between these ecological situations and the simple model studied in this paper. Firstly, the systems are at some distance from Hopf bifurcation; indeed, any population cycles that merit the time and expense of spatiotemporal field study will inevitably be of relatively large amplitude. Secondly, although the Robin/Dirichlet boundary conditions are relative to zero population density in both ecological systems and the $\lambda-\omega$ model, in the former case the cycles occur around a different, non-trivial equilibrium. These differences mean that a significant extension of my work is needed before a full comparison of Robin and Dirichlet boundary conditions can be made in realistic ecological models. 
There is an increasing body of evidence from ecological field studies suggesting that periodic travelling waves are a widespread spatiotemporal pattern in cyclic populations (Ranta \& Kaitala, 1997; Lambin et al., 1998; Ranta et al., 2002; Johnson et al., 2004; Sherratt \& Smith, 2008). A thorough mathematical understanding of the dynamics underlying such patterns is crucial, in view of the considerable time and expense required for field studies. The work in this paper represents one step in this on-going process.

\section{Acknowledgement}

The work in this paper was motivated by a question asked by Jim Keener (University of Utah) at the 2003 Annual Meeting of The Society of Mathematical Biology and by a subsequent e-mail from Nick Britton (University of Bath). I am very grateful to them both. I am also indebted to Adri Olde Daalhuis (University of Edinburgh) for help with the hypergeometric function and to John Byatt-Smith (University of Edinburgh) for helpful discussions, and for encouragement with the numerical calculations shown in Fig. 3.

\section{Funding}

Advanced Research Fellowship from Engineering and Physical Sciences Research Council.

\section{REFERENCES}

Abramowitz, M. \& Stegun, I. A. (1964) Handbook of Mathematical Functions. New York: Dover.

Auchmuty, J. F. G. \& Nicolis, G. (1976) Bifurcation analysis of reaction-diffusion equations-III. Chemical oscillations. Bull. Math. Biol., 38, 325-350.

Bierman, S. M., Fairbairn, J. P., Petty, S. J., Elston, D. A., Tidhar, D. \& Lambin, X. (2006) Changes over time in the spatiotemporal dynamics of cyclic populations of field voles (Microtus agrestis L.). Am. Nat., 167, 583-590.

Bjørnstad, O. N., Peltonen, M., Liebhold, A. M. \& Baltensweiler, W. (2002) Waves of larch budmoth outbreaks in the European Alps. Science, 298, 1020-1023.

CAntrell, R. S., Cosner, C. \& FAGAn, W. (1998) Competitive reversals inside ecological reserves: the role of external habitat degradation. J. Math. Biol., 37, 491-533.

CANTrell, R. S., Cosner, C. \& FAGAN, W. F. (2002) Habitat edges and predator-prey interactions: effects on critical patch size. Math. Biosci., 175, 31-55.

COPE, D. (1979) Reaction-diffusion systems with explicit traveling wave and transient solutions. SIAM J. Appl. Math., 37, 316-324.

Dillon, R., Maini, P. K. \& OThmeR, H. G. (1994) Pattern formation in generalized Turing systems. 1. Steady state patterns in systems with mixed boundary conditions. J. Math. Biol., 32, 345-393.

Ermentrout, G. B. (1981) Stable small amplitude solutions in reaction-diffusion systems. Q. Appl. Math., 39, 61-86.

Ermentrout, G. B., Chen, X. \& Chen, Z. (1997) Transition fronts and localised structures in bistable reactiondiffusion equations. Physica $D, \mathbf{1 0 8}, 147-167$.

Ermentrout, G. B. \& Kopell, N. (1984) Frequency plateaus in a chain of weakly coupled oscillators. SIAM J. Math. Anal., 15, 215-237.

Ermentrout, G. B. \& Kopell, N. (1986) Symmetry and phase-locking in chains of weakly coupled oscillators. Commun. Pure Appl. Anal., 49, 623-660.

GARVIE, M. R. (2007) Finite difference schemes for reaction-diffusion equations modeling predator-prey interactions in MATLAB. Bull. Math. Biol., 69, 931-956. 
Golubitsky, M., KNObloch, E. \& STEWART, I. (2000) Target patterns and spirals in planar reaction-diffusion systems. J. Nonlinear Sci., 10, 333-354.

Gradshteyn, I. S. \& RyzhiK, I. M. (2000) Table of Integrals, Series, and Products, 6th edn. San Diego, CA: Academic Press.

Guckenheimer, J. \& Holmes, P. (1983) Nonlinear Oscillations, Dynamical Systems and Bifurcations of Vector Fields. Berlin: Springer.

Hagan, P. S. (1981) Target patterns in reaction-diffusion systems. Adv. Appl. Math., 2, 400-416.

Hassan, S. A., Zanette, D. H. \& Wio, H. S. (1994) Stationary states in a reaction-diffusion system with albedo boundary conditions. J. Phys. A, 27, 5129-5134.

Hassard, B. D., KaZarinoff, N. D. \& Wan, Y.-H. (1981) Theory and Applications of Hopf Bifurcation. Cambridge: Cambridge University Press.

Hindmarsh, A. C. (1983) ODEPACK, a systematized collection of ODE solvers. Scientific Computing (R. S. Stepleman et al. eds). Amsterdam: North-Holland, pp. 55-64.

IZUS, G. G., DE RUEDA, J. R. \& BORZI, C. H. (1998) Boundary effects on the structural stability of stationary patterns in a bistable reaction-diffusion system. J. Stat. Phys., 90, 103-117.

Johnson, D. M., BJøRnstad, O. N. \& Liebhold, A. M. (2004) Landscape geometry and travelling waves in the larch budmoth. Ecol. Lett., 7, 967-974.

KaPitula, T. (1994) On the nonlinear stability of plane waves for the Ginzburg-Landau equation. Commun. Pure Appl. Math., 47, 831-841.

KAY, A. L. \& Sherratt, J. A. (2000) Spatial noise stabilizes periodic wave patterns in oscillatory systems on finite domains. SIAM J. Appl. Math., 61, 1013-1041.

KopelL, N. (1981) Target pattern solutions to reaction-diffusion equations in the presence of impurities. Adv. Appl. Math., 2, 389-399.

Kopell, N., Ermentrout, G. B. \& Williams, T. L. (1991) On chains of oscillators forced at one end. SIAM J. Appl. Math., 51, 1397-1417.

Kopell, N. \& Howard, L. N. (1973) Plane wave solutions to reaction-diffusion equations. Stud. Appl. Math., 52, 291-328.

Lambin, X., Elston, D. A., Petty, S. J. \& Mackinnon, J. L. (1998) Spatial asynchrony and periodic travelling waves in cyclic populations of field voles. Proc. R. Soc. Lond. B, 265, 1491-1496.

Ludwig, D., Aronson, D. G. \& Weinberger, H. F. (1979) Spatial patterning of the spruce budworm. J. Math. Biol., 8, 217-258.

MacKinnon, J. L., Lambin, X., Elston, D. A., Thomas, C. J., Sherratt, T. N. \& Petty, S. J. (2001) Scale invariant spatio-temporal patterns of field vole density. J. Anim. Ecol., 70, 101-111.

MAGINU, K. (1979) Stability of spatially homogeneous periodic solutions of reaction-diffusion equations. J. Differ. Equ., 31, 130-138.

MAGINU, K. (1981) Stability of periodic travelling wave solutions with large spatial periods in reaction-diffusion systems. J. Differ. Equ., 39, 73-99.

Maini, P. K., WeI, J. C. \& Winter, M. (2007) Stability of spikes in the shadow Gierer-Meinhardt system with Robin boundary conditions. Chaos, 17, 037106.

Medvinsky, A. B., Petrovski, S. V., Tikhonova, I. A., Malchow, H. \& Li, B.-L. (2002) Spatiotemporal complexity of plankton and fish dynamics. SIAM Rev., 44, 311-370.

Moss, R., Elston, D. A. \& WATson, A. (2000) Spatial asynchrony and demographic travelling waves during red grouse population cycles. Ecology, 81, 981-989.

Pascual, M. (1993) Diffusion induced chaos in a spatial predator-prey system. Proc. R. Soc. Lond. B, 251, $1-7$.

Petrovskit, S. V., Kawasaki, K., TAkasu, F. \& Shigesada, N. (2001) Diffusive waves, dynamical stabilization and spatio-temporal chaos in a community of three competitive species. Jpn. J. Ind. Appl. Math., 18, 459-481. 
Petrovskit, S. V. \& MAlchow, H. (2000) Critical phenomena in plankton communities: KISS model revisited. Nonlinear Anal. Real World Appl., 1, 37-51.

Petrovskit, S. V. \& Malchow, H. (2001) Wave of chaos: new mechanism of pattern formation in spatiotemporal population dynamics. Theor. Popul. Biol., 59, 157-174.

Petrovskit, S. V., Vinogradov, M. E. \& Morozov, A. Y. (1998) Spatial-temporal dynamics of a localized populational burst in a distributed prey-predator system. Okeanologiya, 38, 881-890.

Petty, S. J., Lambin, X., Sherratt, T. N., Thomas, C. J., MacKinnon, J. L., Coles, C. F., Davison, M. \& LiTTLE, B. (2000) Spatial synchrony in field vole Microtus agrestis abundance in a coniferous forest in northern England: the role of vole-eating raptors. J. Appl. Ecol., 37, 136-147.

Petzold, L. R. (1983) Automatic selection of methods for solving stiff and nonstiff systems of ordinary differential equations. SIAM J. Sci. Stat. Comput., 4, 136-148.

Piertney, S. B., MacColl, A. D. C., Bacon, P. J. \& Dallas, J. F. (1998) Local genetic structure in red grouse (Lagopus lagopus scoticus): evidence from microsatellite DNA markers. Mol. Ecol., 7, $1645-1654$.

Polyanin, A. D. \& Zaitsev, V. F. (2003) Handbook of Exact Solutions for Ordinary Differential Equations, 2nd edn. Boca Raton, FL: CRC Press.

RAMOS, J. I. (2003) Spatio-temporal patterns in two-dimensional excitable media subject to Robin boundary conditions. Appl. Math. Comput., 146, 55-72.

Ranta, E., Lundberg, P., Kaitala, V. \& Stenseth, N. C. (2002) On the crest of a population wave. Science, 298, 973-974.

Ranta, E. \& Kaitala, V. (1997) Travelling waves in vole population dynamics. Nature, 390, 456.

Ren, L. \& ERmentrout, G. B. (1998) Monotonicity of phaselocked solutions in chains and arrays of nearestneighbor coupled oscillators. SIAM J. Math. Anal., 29, 208-234.

Romero, J. L., Gandarias, M. L. \& Medina, E. (2000) Symmetries, periodic plane waves and blow-up of lambda-omega systems. Physica D, 147, 259-272.

SHERRATT, J. A. (1994) On the evolution of periodic plane waves in reaction-diffusion systems of $\lambda-\omega$ type. SIAM J. Appl. Math., 54, 1374-1385.

SHERRATT, J. A. (1996) Oscillatory and chaotic wakes behind moving boundaries in reaction-diffusion systems. Dyn. Stab. Syst., 11, 303-324.

Sherratt, J. A. (2001) Periodic travelling waves in cyclic predator-prey systems. Ecol. Lett., 4, 30-37.

SHERRATT, J. A. (2003) Periodic travelling wave selection by Dirichlet boundary conditions in oscillatory reactiondiffusion systems. SIAM J. Appl. Math., 63, 1520-1538.

Sherratt, J. A. \& SMith, M. J. (2008) Periodic travelling waves in cyclic populations: field studies and reactiondiffusion models. J. R. Soc. Interface, 5, 483-505.

Smith, M. J., Sherratt, J. A. \& Armstrong, N. J. (2008) The effects of obstacle size on periodic travelling waves in oscillatory reaction-diffusion equations. Proc. R. Soc. Lond. A, 464, 365-390.

SNEYD, J. \& SHERRATT, J. A. (1997) On the propagation of calcium waves in an inhomogeneous medium. SIAM J. Appl. Math., 57, 73-94.

Timofeeva, Y. \& Coombes, S. (2003) Wave bifurcation and propagation failure in a model of calcium release. J. Math. Biol., 47, 249-269.

von Haeften, B., Izus, G., Deza, R. \& Borzi, C. (1997) Boundary effects on the dynamics of monostable reaction-diffusion systems. Phys. Lett. A, 236, 403-408.

Watson, A., Moss, R., Rothery, P. \& PARr, R. (1984) Demographic causes and predictive models of population fluctuations in red grouse. J. Anim. Ecol., 53, 639-662.

WEBB, S. D. \& SHERRATT, J. A. (2004) Oscillatory reaction-diffusion equations with temporally varying parameters. Math. Comp. Modelling, 39, 45-60.

Wio, H. S., IzUs, G., Ramirez, O., Deza, R. \& Borzi, C. (1993) Pattern formation in an activator inhibitor model—effect of albedo boundary conditions on a finite geometry. J. Phys. A, 26, 4281-4286. 


\section{Appendix A}

\section{A.1 Introduction}

In this appendix, I discuss the behaviour as $y \rightarrow \infty$ of the general solutions of (16) and (30). Recall from (22) and (31) that these are given by the sum $C_{1, i} \mathcal{T}_{1}+C_{2, i} \mathcal{T}_{2}+C_{3, i} \mathcal{T}_{3}+\mathcal{T}_{4, i}$, where $C_{1, i}, C_{2, i}$ and $C_{3, i}$ are constants of integration and

$$
\begin{aligned}
& \mathcal{T}_{1}=\operatorname{sech}^{2} y \int_{y_{1}=0}^{y_{1}=y} Y^{+}\left(y_{1}\right) \mathrm{d} y_{1}, \\
& \mathcal{T}_{2}=\operatorname{sech}^{2} y \int_{y_{1}=0}^{y_{1}=y} Y^{-}\left(y_{1}\right) \mathrm{d} y_{1}, \\
& \mathcal{T}_{3}=\operatorname{sech}^{2} y, \\
& \mathcal{T}_{4, i}=K_{w} \operatorname{sech}^{2} y\left[\int_{y_{1}=0}^{y_{1}=y} Y^{-}\left(y_{1}\right) \int_{y_{2}=y_{1}}^{y_{2}=\infty} \frac{Y^{+}\left(y_{2}\right) g_{i}\left(y_{2}\right)}{\cosh ^{4} y_{2}} \mathrm{~d} y_{2} \mathrm{~d} y_{1}\right. \\
& \left.\quad-\int_{y_{1}=0}^{y_{1}=y} Y^{+}\left(y_{1}\right) \int_{y_{2}=y_{1}}^{y_{2}=\infty} \frac{Y^{-}\left(y_{2}\right) g_{i}\left(y_{2}\right)}{\cosh ^{4} y_{2}} \mathrm{~d} y_{2} \mathrm{~d} y_{1}\right] .
\end{aligned}
$$

Here, $Y^{ \pm}(y)=\operatorname{Re}\left[\operatorname{sech}^{p} y \mathcal{F}\left(\alpha, \beta, \gamma, \frac{1 \pm \tanh y}{2}\right)\right], p=-3+\sqrt{12 a^{2}-11}, \alpha+\beta=1+2 \sqrt{12 a^{2}-11}$ and $\alpha \beta=\gamma=1+\sqrt{12 a^{2}-11}$. The constant $K_{w}$ is defined by $Y^{+}(y) Y^{-^{\prime}}(y)-Y^{-}(y) Y^{+^{\prime}}(y)=$ $K_{w} \cosh ^{6} y$. By construction, $\mathcal{T}_{4, i} \rightarrow A_{i}$ as $y \rightarrow \infty$, and the limiting behaviour $\mathcal{T}_{3} \sim 4 \mathrm{e}^{-2 y}$ as $y \rightarrow \infty$ is immediate. However, the behaviour of $\mathcal{T}_{1}$ and $\mathcal{T}_{2}$ as $y \rightarrow \infty$ depends on $\gamma-\alpha-\beta=-\sqrt{12 a^{2}-11}$, and I consider separately the three cases of $a^{2}$ greater than, equal to and less than $11 / 12$.

A.2 Case 1: $1>a^{2}>11 / 12$

A.2.1 Leading-order behaviour of $\mathcal{T}_{1}$. For values of $a$ in this range, $\gamma-\alpha-\beta$ is real and negative, so that

$$
F(\alpha, \beta, \gamma ; \xi) \sim \frac{\Gamma(\gamma) \Gamma(\alpha+\beta-\gamma)}{\Gamma(\alpha) \Gamma(\beta)}(1-\xi)^{\gamma-\alpha-\beta} \quad \text { as } \xi \rightarrow 1^{-}
$$

(Abramowitz \& Stegun, 1964, Section 15.3.6). Therefore, as $y \rightarrow \infty$

$$
\begin{aligned}
Y^{+}(y) & \sim \frac{\Gamma(\gamma) \Gamma(\alpha+\beta-\gamma)}{\Gamma(\alpha) \Gamma(\beta)}\left(\mathrm{e}^{-2 y}\right)^{\gamma-\alpha-\beta}\left(2 \mathrm{e}^{-y}\right)^{p} \\
& =\frac{2^{p} \Gamma(\gamma) \Gamma(\alpha+\beta-\gamma)}{\Gamma(\alpha) \Gamma(\beta)} \exp \left\{\left(3+\sqrt{12 a^{2}-11}\right) y\right\} .
\end{aligned}
$$

Here, I am using $p=-3+\sqrt{12 a^{2}-11}$. Therefore,

$$
\mathcal{T}_{1} \sim \frac{2^{p+2} \Gamma(\gamma) \Gamma(\alpha+\beta-\gamma)}{\Gamma(\alpha) \Gamma(\beta)\left(3+\sqrt{12 a^{2}-11}\right)} \exp \left\{\left(1+\sqrt{12 a^{2}-11}\right) y\right\} \quad \text { as } y \rightarrow \infty .
$$


A.2.2 Leading-order behaviour of $\mathcal{T}_{2} . \quad F(\alpha, \beta, \gamma ; 0)=1$ in all cases (Abramowitz \& Stegun, 1964, Section 15.1.1). Therefore, $Y^{-}(y) \sim 2^{p} \mathrm{e}^{-p y}$ as $y \rightarrow \infty$ and thus

$$
\mathcal{T}_{2} \sim \frac{2^{p+2}}{3-\sqrt{12 a^{2}-11}} \exp \left\{\left(1-\sqrt{12 a^{2}-11}\right) y\right\} \quad \text { as } y \rightarrow \infty .
$$

A.2.3 Conclusions in this case. From these various leading-order forms, it follows that $\mathcal{T}_{1}$ and $\mathcal{T}_{2} \rightarrow$ $\infty$ as $y \rightarrow \infty$, at different rates, while $\mathcal{T}_{3} \rightarrow 0$ and $\mathcal{T}_{4, i} \rightarrow A_{i}$. Since the sum $C_{1, i} \mathcal{T}_{1}+C_{2, i} \mathcal{T}_{2}+$ $C_{3, i} \mathcal{T}_{3}+\mathcal{T}_{4, i}$ must approach a finite limit as $y \rightarrow \infty$, it is necessary that $C_{1, i}=C_{2, i}=0$.

\section{A.3 Case 2: $a^{2}=11 / 12$}

A.3.1 Leading-order form of $\mathcal{T}_{1}$. When $a^{2}=11 / 12, \gamma-\alpha-\beta=0$, so that

$$
F(\alpha, \beta, \gamma ; \xi) \sim \frac{-\Gamma(\alpha+\beta) \log (1-\xi)}{\Gamma(\alpha) \Gamma(\beta)} \text { as } \xi \rightarrow 1^{-}
$$

(Abramowitz \& Stegun, 1964, Section 15.3.10). Therefore, as $y \rightarrow \infty$,

$$
Y^{+}(y) \sim \frac{y \mathrm{e}^{3 y}}{4 \Gamma(\alpha) \Gamma(\beta)} .
$$

Here, I am using the fact that $\alpha+\beta=1$ and $p=-3$ in this case. Therefore,

$$
\mathcal{T}_{1} \sim \frac{y \mathrm{e}^{y}}{3 \Gamma(\alpha) \Gamma(\beta)} \quad \text { as } y \rightarrow \infty
$$

A.3.2 Leading-order form of $\mathcal{T}_{2} \quad$ As in Section A.2.2, $\mathcal{T}_{2} \sim \frac{1}{6} \mathrm{e}^{y}$ as $y \rightarrow \infty$.

A.3.3 Conclusions in this case. Again $\mathcal{T}_{1}$ and $\mathcal{T}_{2} \rightarrow \infty$ at different rates as $y \rightarrow \infty$, while $\mathcal{T}_{3} \rightarrow 0$ and $\mathcal{T}_{4, i} \rightarrow A_{i}$. Since the sum $C_{1, i} \mathcal{T}_{1}+C_{2, i} \mathcal{T}_{2}+C_{3, i} \mathcal{T}_{3}+\mathcal{T}_{4, i}$ must approach a finite limit as $y \rightarrow \infty$, it is necessary that $C_{1, i}=C_{2, i}=0$.

A.4 Case 3: $a^{2}<11 / 12$

A.4.1 Leading-order form of $\mathcal{T}_{1}$. When $a^{2}<11 / 12, \gamma-\alpha-\beta$ is pure imaginary, so that

$$
F(\alpha, \beta, \gamma ; \xi) \sim \frac{\Gamma(\gamma) \Gamma(\gamma-\alpha-\beta)}{\Gamma(\gamma-\alpha) \Gamma(\gamma-\beta)}+\frac{\Gamma(\gamma) \Gamma(\alpha+\beta-\gamma)}{\Gamma(\alpha) \Gamma(\beta)}(1-\xi)^{\gamma-\alpha-\beta} \quad \text { as } \xi \rightarrow 1^{-}
$$

(Abramowitz \& Stegun, 1964, Section 15.3.6). Therefore,

$$
\begin{aligned}
& \operatorname{sech}^{p} y F\left(\alpha, \beta, \gamma, \frac{1+\tanh y}{2}\right) \sim {\left[\frac{\Gamma(\gamma) \Gamma(\gamma-\alpha-\beta)}{\Gamma(\gamma-\alpha) \Gamma(\gamma-\beta)}+\frac{\Gamma(\gamma) \Gamma(\alpha+\beta-\gamma)}{\Gamma(\alpha) \Gamma(\beta)} \mathrm{e}^{2(\alpha+\beta-\gamma) y}\right] 2^{p} \mathrm{e}^{-p y} } \\
&= \frac{\Gamma(\gamma) \Gamma(\gamma-\alpha-\beta)}{\Gamma(\gamma-\alpha) \Gamma(\gamma-\beta)} 2^{p} \exp \left\{\left(3-\sqrt{12 a^{2}-11}\right) y\right\} \\
&+\frac{\Gamma(\gamma) \Gamma(\alpha+\beta-\gamma)}{\Gamma(\alpha) \Gamma(\beta)} 2^{p} \exp \left\{\left(3+\sqrt{12 a^{2}-11}\right) y\right\} \quad \text { as } y \rightarrow \infty \\
& \Rightarrow Y^{+}(y) \equiv \operatorname{Re}\left[\operatorname{sech}^{p} y F\left(\alpha, \beta, \gamma, \frac{1+\tanh y}{2}\right)\right] \sim\left(k_{1} \sin \delta y+k_{2} \cos \delta y\right) \mathrm{e}^{3 y} \quad \text { as } y \rightarrow \infty .
\end{aligned}
$$


Here, the real numbers $\delta, k_{1}$ and $k_{2}$ are given by

$$
\begin{gathered}
\delta=-\mathrm{i} \sqrt{12-11 a^{2}} \\
k_{1}=\operatorname{Im}\left[2^{p} \Gamma(\gamma)\left\{\frac{\Gamma(\alpha+\beta-\gamma)}{\Gamma(\alpha) \Gamma(\beta)}-\frac{\Gamma(\gamma-\alpha-\beta)}{\Gamma(\gamma-\alpha) \Gamma(\gamma-\beta)}\right\}\right], \\
k_{2}=\operatorname{Re}\left[2^{p} \Gamma(\gamma)\left\{\frac{\Gamma(\alpha+\beta-\gamma)}{\Gamma(\alpha) \Gamma(\beta)}+\frac{\Gamma(\gamma-\alpha-\beta)}{\Gamma(\gamma-\alpha) \Gamma(\gamma-\beta)}\right\}\right] .
\end{gathered}
$$

Therefore,

$$
\mathcal{T}_{1} \sim \frac{4 \mathrm{e}^{y}}{9+\delta^{2}}\left[\left(3 k_{1}+\delta k_{2}\right) \sin \delta y+\left(3 k_{2}-\delta k_{1}\right) \cos \delta y\right] \quad \text { as } y \rightarrow \infty
$$

\section{A.4.2 Leading-order behaviour of $\mathcal{T}_{2}$. As in Section A.2.2,}

$$
Y^{-}(y) \sim \operatorname{Re}\left[2^{p} \mathrm{e}^{-p y}\right]=\left(k_{3} \sin \delta y+k_{4} \cos \delta y\right) \mathrm{e}^{3 y} \quad \text { as } y \rightarrow \infty,
$$

where $k_{3}=-\operatorname{Im}\left(2^{p}\right)$ and $k_{4}=\operatorname{Re}\left(2^{p}\right)$. Therefore,

$$
\mathcal{T}_{2} \sim \frac{4 \mathrm{e}^{y}}{9+\delta^{2}}\left[\left(3 k_{3}+\delta k_{4}\right) \sin \delta y+\left(3 k_{4}-\delta k_{3}\right) \cos \delta y\right] \quad \text { as } y \rightarrow \infty .
$$

A.4.3 Conclusion in this case. Again $\mathcal{T}_{1}$ and $\mathcal{T}_{2} \rightarrow \infty$ as $y \rightarrow \infty$, while $\mathcal{T}_{3} \rightarrow 0$ and $\mathcal{T}_{4, i} \rightarrow A_{i}$. In this case, $\mathcal{T}_{1}$ and $\mathcal{T}_{2}$ exhibit growing oscillations as $y \rightarrow \infty$, with the same growth rate, but their linear independence implies that $C_{1, i}=C_{2, i}=0$.

\section{Appendix B}

In this appendix, I derive three results which together imply that $I_{1}=I_{2}=0$.

\section{B.1 First result}

I begin by showing that

$$
\int_{-\infty}^{+\infty} F\left(\alpha, \beta, \gamma ; \frac{1+\tanh y}{2}\right) \operatorname{sech}^{10+p} y \mathrm{~d} y=\frac{6-2 a^{2}}{7-2 a^{2}} \int_{-\infty}^{+\infty} F\left(\alpha, \beta, \gamma ; \frac{1+\tanh y}{2}\right) \operatorname{sech}^{8+p} y \mathrm{~d} y .
$$

I define

$$
Q_{\mu}=\int_{-\infty}^{+\infty} F\left(\alpha, \beta, \gamma ; \frac{1+\tanh y}{2}\right) \operatorname{sech}^{2 \mu} y \mathrm{~d} y
$$

Substituting $\xi=(1+\tanh y) / 2$ in the integral gives

$$
Q_{\mu}=2^{2 \mu-3} \int_{0}^{1}\left(\xi-\xi^{2}\right)^{\mu-1} F(\alpha, \beta, \gamma ; \xi) \mathrm{d} \xi
$$


From (19),

$$
F(\alpha, \beta, \gamma ; \xi)=\frac{\xi-\xi^{2}}{p+4} \frac{\mathrm{d}^{2} F}{\mathrm{~d} \xi^{2}}+(1-2 \xi) \frac{\mathrm{d} F}{\mathrm{~d} \xi}
$$

here and henceforth, I omit the arguments of $F$ for brevity. Substituting (B.3) into (B.2) gives

$$
\begin{aligned}
Q_{\mu} & =\frac{2^{2 \mu-3}}{p+4} \int_{0}^{1}\left(\xi-\xi^{2}\right)^{\mu} \frac{\mathrm{d}^{2} F}{\mathrm{~d} \xi^{2}} \mathrm{~d} \xi+2^{2 \mu-3} \int_{0}^{1}\left(\xi-\xi^{2}\right)^{\mu-1}(1-2 \xi) \frac{\mathrm{d} F}{\mathrm{~d} \xi} \mathrm{d} \xi \\
& =2^{2 \mu-3}\left(1-\frac{\mu}{p+4}\right) \int_{0}^{1}\left(\xi-\xi^{2}\right)^{\mu-1}(1-2 \xi) \frac{\mathrm{d} F}{\mathrm{~d} \xi} \mathrm{d} \xi
\end{aligned}
$$

on integrating the first integral by parts. Applying integration by parts again gives

$$
\begin{aligned}
Q_{\mu} & =2^{2 \mu-3}\left(1-\frac{\mu}{p+4}\right) \int_{0}^{1} F(\xi)\left[2\left(\xi-\xi^{2}\right)^{\mu-1}+(1-\mu)\left(\xi-\xi^{2}\right)^{\mu-2}(1-2 \xi)^{2}\right] \mathrm{d} \xi \\
& =\left(1-\frac{\mu}{p+4}\right)\left\{(4 \mu-2) Q_{\mu}+4(1-\mu) Q_{\mu-1}\right\} \\
\Rightarrow Q_{\mu} & =\frac{4(p+4-\mu)(1-\mu)}{(p+4)(3-4 \mu)+2 \mu(2 \mu-1)} Q_{\mu-1} .
\end{aligned}
$$

Substituting $\mu=5+p / 2$ and $p=-3+\sqrt{12 a^{2}-11}$ into (B.4) gives (B.1).

\section{B.2 Second result}

I now show that $\operatorname{Re} J \neq 0$ for all $a \in(0,1)$, where

$$
J=\int_{y=0}^{y=\infty}\left[F\left(\alpha, \beta, \gamma ; \frac{1+\tanh y}{2}\right)+F\left(\alpha, \beta, \gamma ; \frac{1-\tanh y}{2}\right)\right] \tanh ^{2} y \operatorname{sech}^{4+p} y \mathrm{~d} y .
$$

Straightforward simplification shows that $J=Q_{2+p / 2} / 2^{1+p}-Q_{3+p / 2} / 2^{3+p}$, and (B.4) then gives

$$
J=\frac{3 p^{2}+18 p+16}{2^{p+3}\left(p^{2}+6 p+6\right)} Q_{2+p / 2}=2^{1-\sqrt{12 a^{2}-11}} \frac{2+9\left(1-a^{2}\right)}{1+6\left(1-a^{2}\right)} Q_{2+p / 2} .
$$

Because $\gamma=(1+\alpha+\beta) / 2$, the hypergeometric function $F(\alpha, \beta, \gamma ; z)$ can be expressed in terms of an associated Legendre function of the first kind (see Abramowitz \& Stegun, 1964, Section 15.4.19). This implies

$$
\begin{aligned}
Q_{2+p / 2} & =2^{p+2} \Gamma\left(\frac{1+\alpha+\beta}{2}\right) \int_{-1}^{+1}(1-\zeta)^{-1 / 2} P_{(\alpha-\beta-1) / 2}^{(1-\alpha-\beta) / 2}(\zeta) \mathrm{d} \zeta \\
& =\frac{2^{p+2+(1-\alpha-\beta) / 2} \pi \Gamma\left(\frac{3-\alpha-\beta}{4}\right) \Gamma\left(\frac{1+\alpha+\beta}{4}\right) \Gamma\left(\frac{1+\alpha+\beta}{2}\right)}{\Gamma\left(\frac{3+\alpha-\beta}{4}\right) \Gamma\left(\frac{3-\alpha+\beta}{4}\right) \Gamma\left(\frac{1+\alpha}{2}\right) \Gamma\left(\frac{1+\beta}{2}\right)}
\end{aligned}
$$


using Gradshteyn \& Ryzhik (2000, Section 7.132.1). Explicit calculation of $\alpha$ and $\beta$ using (21) then gives

$$
J=\frac{\pi \frac{2+9\left(1-a^{2}\right)}{2+12\left(1-a^{2}\right)} \Gamma\left(\frac{1-\sqrt{12 a^{2}-11}}{2}\right) \Gamma\left(\frac{1+\sqrt{12 a^{2}-11}}{2}\right) \Gamma\left(1+\sqrt{12 a^{2}-11}\right)}{\Gamma\left(\frac{3-\sqrt{48 a^{2}-47}}{4}\right) \Gamma\left(\frac{3+\sqrt{48 a^{2}-47}}{4}\right) \Gamma\left(\frac{3+2 \sqrt{12 a^{2}-11}-\sqrt{48 a^{2}-47}}{4}\right) \Gamma\left(\frac{3+2 \sqrt{12 a^{2}-11}+\sqrt{48 a^{2}-47}}{4}\right)} .
$$

All the various arguments of the Gamma functions in this expression have positive real part whenever $a \in(0,1)$, and therefore $J \neq 0$.

It remains to show that $J$ is not pure imaginary. The expressions $\left(1 \pm \sqrt{12 a^{2}-11}\right) / 2$ are either real or complex conjugate, and since $\Gamma(\bar{z})=\overline{\Gamma(z)}$, the product $\Gamma\left(\frac{1-\sqrt{12 a^{2}-11}}{2}\right) \Gamma\left(\frac{1+\sqrt{12 a^{2}-11}}{2}\right)$ is always real. Similar reasoning shows that $J$ is always real if $a>\sqrt{11 / 12}$; otherwise,

$$
\arg J=\arg \Gamma(1+\mathrm{i} \sigma)-\arg \Gamma\left(\frac{3+2 \mathrm{i} \sigma+\mathrm{i} \sqrt{3+4 \sigma^{2}}}{4}\right)-\arg \Gamma\left(\frac{3+2 \mathrm{i} \sigma-\mathrm{i} \sqrt{3+4 \sigma^{2}}}{4}\right),
$$

where $\sigma=\sqrt{11-12 a^{2}}$. Combining Sections 6.1.27, 6.3.2 and 6.3.16 in Abramowitz \& Stegun (1964) then gives $\arg J=\sum_{n=0}^{n=\infty} q_{n}$, where

$$
q_{n}=\tan ^{-1}\left(\frac{2 \sigma+\sqrt{3+4 \sigma^{2}}}{4 n+3}\right)+\tan ^{-1}\left(\frac{2 \sigma-\sqrt{3+4 \sigma^{2}}}{4 n+3}\right)-\tan ^{-1}\left(\frac{\sigma}{n+1}\right) .
$$

Explicit differentiation gives

$$
\frac{\mathrm{d}^{2} q_{n}}{\mathrm{~d} \sigma^{2}}=\frac{2 \sigma n Q_{n}\left(\sigma^{2}\right)}{\left[\left(4 n^{2}+6 n+3+2 \sigma^{2}\right)^{2}-\sigma^{2}\left(3+4 \sigma^{2}\right)\right]^{2}\left(n^{2}+2 n+1+\sigma^{2}\right)^{2}} .
$$

Here, $Q_{n}\left(\sigma^{2}\right)$ is a quadratic function of $\sigma^{2}$, and explicit calculation of the roots of $Q_{n}$ shows that $Q_{n}\left(\sigma^{2}\right)<0$ for all $n \geqslant 1$ and $\sigma \in(0, \sqrt{11})$ (details omitted for brevity). Therefore, $\mathrm{d}^{2} q_{n} / \mathrm{d} \sigma^{2}<$ 0 for all $n \geqslant 1$ and $\sigma \in(0, \sqrt{11})$. Straightforward applications of the Weierstrass test show that $\sum_{n=0}^{n=\infty} \mathrm{d} q_{n} / \mathrm{d} \sigma$ and $\sum_{n=0}^{n=\infty} \mathrm{d}^{2} q_{n} / \mathrm{d} \sigma^{2}$ both converge uniformly on $\sigma \in[0, \sqrt{11}]$, and thus $\mathrm{d}^{2} \arg J /$ $\mathrm{d} \sigma^{2}<0$ for all $\sigma \in(0, \sqrt{11})$. Moreover, direct calculation shows that $\mathrm{d} \arg J / \mathrm{d} \sigma>0$ when $\sigma=\sqrt{11}$, and thus $\mathrm{d} \arg J / \mathrm{d} \sigma>0$ for all $\sigma \in(0, \sqrt{11})$. (Note that $\mathrm{d} q_{n} / \mathrm{d} \sigma$ is not positive for all $\sigma$ and $n$.) Finally, $\arg J=0$ when $\sigma=0$ and $\arg J \approx 0.075 \pi$ when $\sigma=\sqrt{11}$, by direct calculation. Therefore, $\arg J \in(0, \pi / 2)$ for all $\sigma \in(0, \sqrt{11})$, i.e. for all $a \in(0,1)$, so that $J$ is never pure imaginary. In combination with the result that $J \neq 0$, this implies that $\operatorname{Re} J \neq 0$ for all $a \in(0,1)$.

\section{B.3 Third result}

Finally, I show that $\operatorname{Re} F^{\prime}\left(\alpha, \beta, \gamma ; \frac{1}{2}\right) \neq 0$ for $a \in(0,1)$. Simplification using Abramowitz \& Stegun (1964, Sections 15.1.24 and 15.2.1) gives

$$
F^{\prime}\left(\alpha, \beta, \gamma ; \frac{1}{2}\right)=\frac{4 \sqrt{\pi} \Gamma(\alpha \beta)}{\Gamma(\alpha / 2) \Gamma(\beta / 2)}=\frac{4 \sqrt{\pi} \Gamma\left(1+\sqrt{12 a^{2}-11}\right)}{\Gamma\left(\frac{1+2 \sqrt{12 a^{2}-11}-\sqrt{48 a^{2}-47}}{4}\right) \Gamma\left(\frac{1+2 \sqrt{12 a^{2}-11}+\sqrt{48 a^{2}-47}}{4}\right)} .
$$


All three arguments of the Gamma functions in this expression have positive real part whenever $a \in$ $(0,1)$. Since $\Gamma(z)$ is analytic and non-zero over the whole complex plane except for simple poles at $z=0,-1,-2, \ldots$, this implies that $F^{\prime}\left(\alpha, \beta, \gamma ; \frac{1}{2}\right) \neq 0$ for $0<a<1$. A similar argument to that above shows that $F^{\prime}\left(\alpha, \beta, \gamma ; \frac{1}{2}\right)$ is not pure imaginary; its argument increases monotonically from zero to about $0.27 \pi$ as $a$ decreases from $\sqrt{11 / 12}$ to zero. There is a slight complication in this case, in that the first term in the series expansion of the argument does not always have a negative second derivative with respect to $\sigma$, but this is easily rectified by considering the sum of the first two terms; the other terms all do have negative second derivative. Therefore, $\operatorname{Re} F^{\prime}\left(\alpha, \beta, \gamma ; \frac{1}{2}\right) \neq 0$ for all $a \in(0,1)$.

\section{Appendix C}

In this appendix, I describe the numerical method used to calculate the dependence of periodic travelling wave amplitude $A$ on $\epsilon$. This is used to generate the results shown in Fig. 3.

My basic approach is to solve (12) numerically. The required solution satisfies end conditions at $y=0$ and $y \rightarrow \infty$ that are given in (13), and in practice it is most convenient to solve backwards in $y$, starting close to the steady state $R=A, R^{\prime}=0, \phi=\sqrt{1-A^{2}}$. Straightforward calculation shows that this equilibrium has a unique stable eigenvector, and I solve (12) as an initial-value problem starting close to the steady state, on the eigenvector corresponding to this stable eigenvalue, with $R$ (and $\phi$ ) slightly below their equilibrium values. (Numerical calculations suggest that for initial values on the eigenvector with $R$ and $\phi$ slightly above the steady-state levels, $R$ and $\phi$ increase towards $\infty$ as $y$ is decreased.) I integrate the equations for decreasing $y$, until $\phi$ becomes zero. The appropriate value of $\epsilon$ can then be read off as $R \sqrt{2} / R^{\prime}$. Note that in this numerical approach, it is not necessary to keep track of the actual values of $y$.

I implement this procedure using the solver DLSODAR (Hindmarsh, 1983; Petzold, 1983) which is part of the ODEPACK collection. The solver automatically switches between an Adams predictorcorrector method and a Gear backward differentiation formula method and automatically stops integration when specified algebraic conditions are satisfied. This makes it straightforward to integrate up to the point at which $\phi$ becomes zero. The numerical code is freely available at www.netlib.org. 